\title{
A Systematic Study of Functional Regulatory Modules by miRNA-miRNA Functional Synergistic Network in Hepatocellular Carcinoma
}

\author{
Xiangjun Kong, Xiaomei Geng, Junrui Zhao, Yuanjia Hu* \\ Institute of Chinese Medical Sciences, State Key Laboratory of Quality Research in Chinese Medicine, \\ University of Macau, Macau, China. \\ * Corresponding author. Tel.: +853 88228507; email: yuanjiahu@umac.mo \\ Manuscript submitted March 14, 2015; accepted May 20, 2015. \\ doi: 10.17706/ijbbb.2015.5.3.184-192
}

\begin{abstract}
Hepatocellular carcinoma (HCC) is one of the most common cancers worldwide. Poor understanding of the pathogenesis mechanism of HCC makes it difficult to be diagnosed and treated. The role of microRNAs (miRNAs) associated with progress and metastasis of HCC is emerging recently. For the purpose of systematically understanding the functional regulatory modules of miRNAs in HCC, this study constructed the miRNA-miRNA functional synergistic network (MFSN) based on KEGG pathway functional enrichment of co-targets collected from differentially expressed miRNAs related to HCC. To further understand the complex cooperative relations of miRNAs, two different synergistic subnetworks were generated to exhibit the effect of synergistic suppression of up-regulated miRNAs and synergistic enhancement of down-regulated miRNAs. Moreover, subnetworks of Focal adhesion, MAPK signaling pathway, Wnt signaling pathway, and TGF-beta signaling pathway were also extracted from MFSN to demonstrate synergistic influence profile of miRNAs in a specific functional pathway.
\end{abstract}

Key words: miRNA-miRNA functional synergistic network, hepatocellular carcinoma (HCC), differentially expressed miRNA, miRNA synergism.

\section{Introduction}

Liver cancer, as one of the most prevalently diagnosed cancers worldwide, is a leading cause of cancer-related death [1]. Accounting for $70 \%-80 \%$ of all primary liver cancer cases, hepatocellular carcinoma (HCC) is often associated with cirrhosis, which can be a consequence of various liver diseases including chronic viral hepatitis B or C infections, nonalcoholic steatohepatitis, autoimmune hepatitis, alcohol abuse, primary biliary cirrhosis, and carcinogens exposure [2], [3]. Surgical resection and whole liver transplantation is the only current choice of HCC treatment, whereas chemotherapeutic interventions are ineffective. The low survival rate motivates comprehensive progresses on molecular mechanisms of HCC, which contributes to the diagnosis and treatment.

MicroRNAs (miRNAs), a class of small non-coding RNAs of 18-25 nucleotides in length, can regulate expression of numerous genes in post-transcription process, by which miRNAs modulate various biological processes, such as cell growth, proliferation, differentiation, apoptosis, and metabolism [4], [5]. Particularly, the involvement of miRNAs in tumors is well established, acting as both tumor suppressors and oncogenes [6]. Identification of miRNA dysregulation in HCC provides important implications for novel diagnostic or 
prognostic markers and constitutes potential treatment targets. For example, miRNA-21 was demonstrated as an oncomir of HCC for its overexpression resulting in marked reductions of PTEN, PDCD4, and RECK expression [7]. High expression of miRNA-15b in HCC tissues may indicate a low risk of HCC recurrence [8]. Other investigations showed the involvement down-regulated miRNAs in cell cycle inhibition, such as miR-34a, miR-199-a-5p, miR-223, and so on [9], [10]. Besides, a mount of miRNAs related to HCC have been found and validated from plenty of basic research including high-throughput experiments [11]. However, the potential mechanism of miRNAs' influence on cancer from genome-wide level is still unclear. Few studies focus on the functional relationship among miRNAs, which is of significance to better understand their complex regulations.

Since complex diseases, especially cancers, are affected by a number of miRNAs, the function regulations of miRNAs could be suggested as synergistic results of differentially expressed miRNAs (DE-miRNAs) related to diseases. It is still a challenge to determine DE-miRNAs' synergistic function at a systematic level related to disease development. Nevertheless, based on aforementioned tremendous evidence from experimental research, it is possible to identify complex synergistic relations among miRNAs. Some research groups have developed some systematic analysis models by constructing miRNA-miRNA functional synergistic network (MFSN), to detect synergistic mechanisms of miRNAs' regulation [12]-[15]. However, potential characteristics of miRNAs synergistic function profile in HCC have been little studied, especially roles of disease-related up- or down-regulated miRNAs, indicating different application potential.

In this study, we attempted to systematically present the miRNA synergism in HCC, via constructing MFSN based on co-targets' functional enrichment of miRNA pair (Fig. 1). Meanwhile, synergistic subnetworks from up- and down-regulated miRNAs, as well as function-based subnetworks are particularly extracted from total MFSN to further examine the mechanisms of miRNA synergism. This research would contribute to primary understanding of miRNAs coordination profile in HCC and further provide evidences for diagnosis and treatment of HCC.

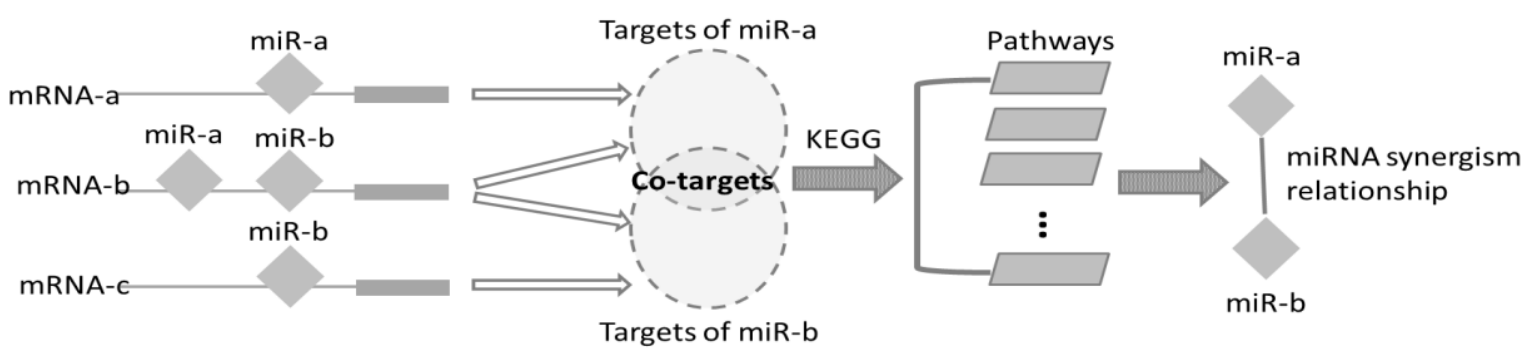

Fig. 1. The workflow to construct the miRNA-miRNA functional synergistic network related to HCC.

\section{Materials and Methods}

\subsection{Data Collection and Processing}

The first step of this study is to retrieve DE-miRNAs related to HCC. We collected DE-miRNAs of HCC from miRCancer database. Established on empirical evidences, miRCancer offers abundant and credible information about miRNA-cancer associations [16]. Thus, a list of HCC relevant miRNAs with expression features was obtained for further target prediction.

Since miRNAs function in post-transcriptional regulation by targeting mRNAs, we studied the role of DE-miRNAs by identifying their putative targets. The potential targets of DE-miRNAs were acquired from miRecords, which was an integrated database to predict miRNA targets, gathering 11 established miRNA target prediction programs containing argetScan, PicTar, DIANA-microT, miRanda, MicroInspector, 
mirTarget2, miTarget, NBmiRTar, PITA, RNA22, and RNAhybrid [17]. For this investigation, genes are assigned to miRNA target sets only if they are predicted by at least four distinct programs, which helps to reduce the number of false positives. Then, co-targets between each miRNA pair were prepared for functional module identification in order to overview synergism among miRNAs.

\section{2. miRNA-miRNA Functional Synergistic Network Construction}

miRNAs involve in the same or similar functions related to a specific complex disease, by regulating common gene sets. It is of significance to understand miRNAs' synergistic relations and further clarify their functions in disease development with a systematic perspective. Based on co-targets of each miRNA pair, functional modules between miRNAs can be disclosed by performing KEGG functional pathway enrichment analysis, which is conducted by clusterProfiler package in $R$ with $P \leq 0.05$ [18]. If a pair of miRNAs owns common targets and the co-targets significantly enrich in at least one KEGG category, we recognize the synergistic effect of the two miRNAs. After assembling all synergistic miRNA pairs, the miRNA-miRNA functional synergistic network is constructed by Gephi software [19], where nodes represent DE-miRNAs with expression profile and edges denote different synergistic relations referring to co-regulated pathways. Degree of a node means the number of edges connecting the node to others. In the MFSN, edges have been weighted by the number of enriched KEGG pathways, reflecting the breadth of synergistic functional modules between miRNAs. The strength of a node in network is the sum of the weights of all links attached to the node.

\subsection{Expression Profile-Based Subnetworks}

ED-miRNAs, containing up- and down- regulation, result in distinct modulation of gene expression. We classify the synergistic effects as three groups according to miRNAs' differential expression profiles: 1) synergistic suppression of two up-regulated miRNAs; 2) synergistic enhancement of two down-regulated miRNAs; 3) conflict effect between an up-regulated miRNA and down-regulated miRNA. Synergistic networks of up- and down-regulated miRNAs are extracted from total MFSN as subnetworks respectively, and their topological analysis is conducted to figure out the synergistic functions of miRNAs in HCC.

\section{4. miRNA Synergism Profiles on Pathways}

To further analyze the synergistic functions of miRNAs in HCC, several important pathways were derived from the total MFSN to construct function-based subnetworks. Edges in a function-based subnetwork depict the specific functional pathway. Nodes represent the synergistic miRNAs involved in this particular pathway.

\section{Results and Discussion}

\subsection{MFSN Analysis}

MFSN of HCC was generated by following the workflow shown in Fig. 1. Potential target genes of 147 DE-miRNAs in HCC have been retrieved from miRecords. Co-targets of miRNA pair were enriched to KEGG pathways, which constituted the functional synergistic relations of miRNAs. Among the 147 DE-miRNAs, 105 miRNAs were finally used to construct MFSN, including 71 down-regulated miRNAs and 34 up-regulated miRNAs. As shown in Fig. 2, red nodes represent up-regulated miRNAs, while blue nodes represent down-regulated miRNAs. The size of nodes is proportional to the node strength in network. Edges denote KEGG pathways enriched by co-targets of miRNA pairs. The thickness of edges indicates the number of synergistic pathways. The hubs with highest strength are let-7c, miR-203, miR-105, miR-20b, and miR-17-5p, which are considered with higher risks when regulation fails, as they influence an amount of other miRNAs and functional pathways. The five hub miRNAs contain three down-regulated miRNAs and 
two up-regulated miRNAs, implying the importance of up-regulated miRNAs despite low share in MFSN. Moreover, let-7 family, miR-181 family, miR-29 family, mir-17 family, and miR-15 family can be well recognized as they connected closer and stronger to their family members than to others. Normally, miRNAs from the same family tend to exert a similar role due to a conserved sequence and structural configuration [20]. There are 100 KEGG pathways enriched between DE-miRNAs of HCC. The top 10 pathways with highest frequency in MFSN have been listed in Table 1. Pathway in cancer is the most widely enriched pathways of coordinated regulation by 96 miRNAs, followed by Focal adhesion, MAPK signaling pathway, and Endocytosis pathway.

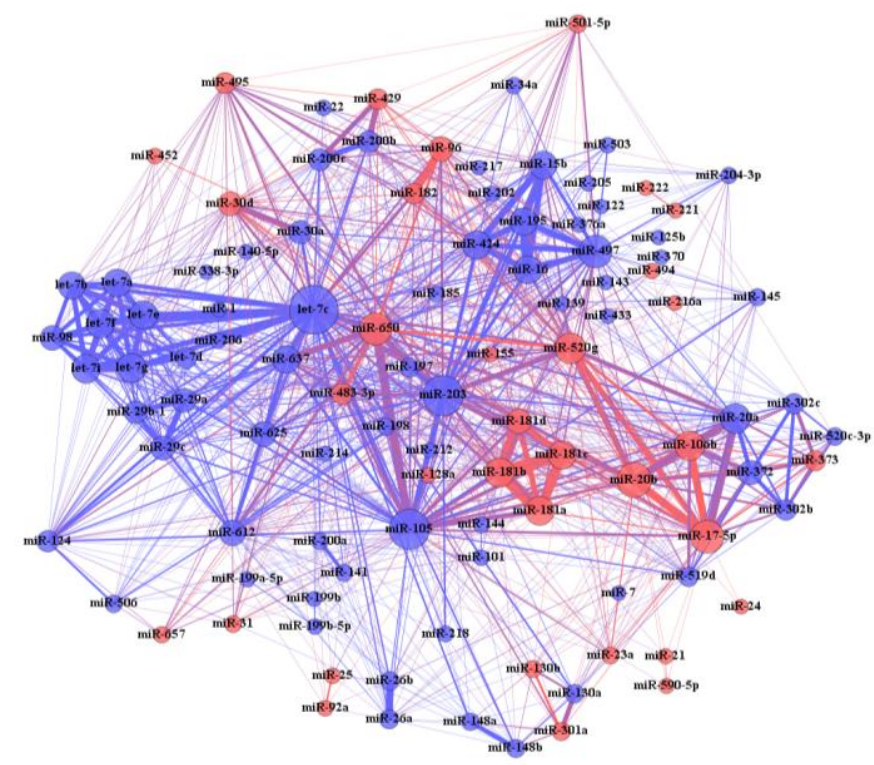

Fig. 2. The miRNA-miRNA functional synergistic network in HCC.

Table 1. Top 10 Pathways with Highest Frequency Involved in the MFSN

\begin{tabular}{|c|c|c|c|c|c|c|c|}
\hline Rank & Pathway ID & Description & Frequency & $\begin{array}{l}\text { No. of involved } \\
\text { miRNAs }\end{array}$ & $\begin{array}{l}\text { Down-regulated } \\
\text { miRNAs }\end{array}$ & $\begin{array}{l}\text { Up-regulated } \\
\text { miRNAs }\end{array}$ & $\mathrm{Ra}^{*}$ \\
\hline 1 & hsa05200 & Pathways in cancer & 590 & 96 & 65 & 31 & 2.1 \\
\hline 2 & hsa 04510 & Focal adhesion & 374 & 75 & 52 & 23 & 2.3 \\
\hline 3 & hsa04010 & MAPK signaling pathway & 276 & 70 & 46 & 24 & 1.9 \\
\hline 4 & hsa04144 & Endocytosis & 268 & 60 & 40 & 20 & 2.0 \\
\hline 5 & hsa04360 & Axon guidance & 229 & 62 & 42 & 20 & 2.1 \\
\hline 6 & hsa04722 & $\begin{array}{l}\text { Neurotrophin signaling } \\
\text { pathway }\end{array}$ & 163 & 40 & 24 & 16 & 1.5 \\
\hline 7 & hsa04810 & $\begin{array}{l}\text { Regulation of actin } \\
\text { cytoskeleton }\end{array}$ & 156 & 69 & 41 & 28 & 1.5 \\
\hline 8 & hsa04310 & Wnt signaling pathway & 149 & 55 & 37 & 18 & 2.1 \\
\hline 9 & hsa04020 & Calcium signaling pathway & 110 & 43 & 27 & 16 & 1.7 \\
\hline 10 & hsa04350 & $\begin{array}{l}\text { TGF-beta signaling } \\
\text { pathway }\end{array}$ & 86 & 42 & 29 & 13 & 2.2 \\
\hline
\end{tabular}

*Ra means the ratio of down-regulated miRNAs to up-regulated miRNAs.

\subsection{Synergistic Subnetwork Generation}

Different expression profile of miRNAs in disease can provide different clinical strategies to target and manipulate either the expression or activity of miRNAs [21]. For example, the oncogenic signaling that promotes tumor development and progression can be repressed by overexpressed miRNAs' regulation, 
which also inactivates associated tumor suppressing genes and pathways. In the MFSN, there are three kind of synergistic relations between miRNAs due to their different expression profiles. Following the classification mentioned above, we extracted two subnetworks to explore miRNA synergism in HCC (Fig. 3). The conflict effect between up- and down-regulated miRNA is out of consideration, as the synergistic outcome on functional pathways could not be determined. The pathways enriched by down-regulated miRNA pairs in Fig. 3(a) are more complex than cooperatively suppressed functions by up-regulated miRNA pairs exhibited in Fig. 3(b). The information of subnetworks is described in Table 2. The average of synergistic suppression is much lower than average synergistic enhancement effect on pathways, detected from the compare of average strength of miRNAs in different subnetworks. Let-7 family members and miR-203 dominate the majority of synergistic connections with most of other down-regulated miRNAs, which could be further investigated for their potential application. Besides, let-7 family, miR-15 family, and miR-29 family function together and assemble the main frame of synergistic enhancement in HCC. Whereas in synergistic suppression network, miR-181 family and mir-17 family occupy the central positon by close connections within family and broad cooperation with other miRNAs.

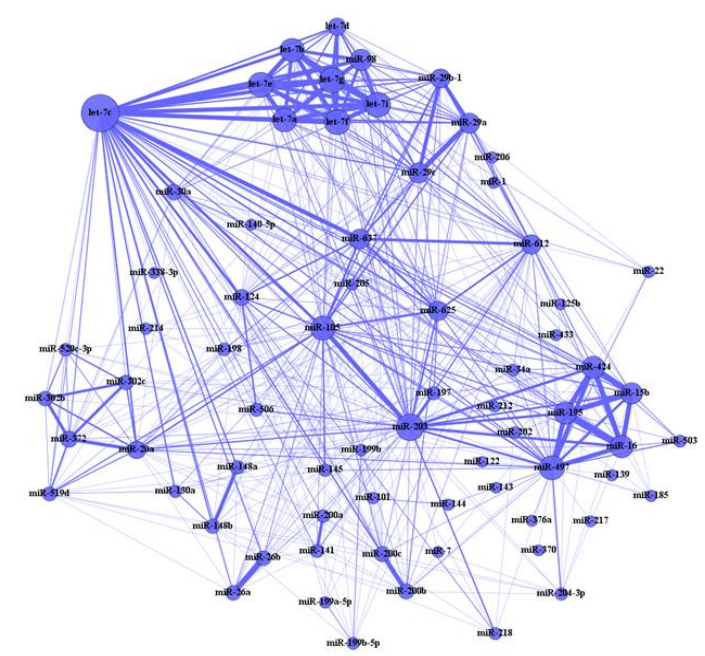

(a)

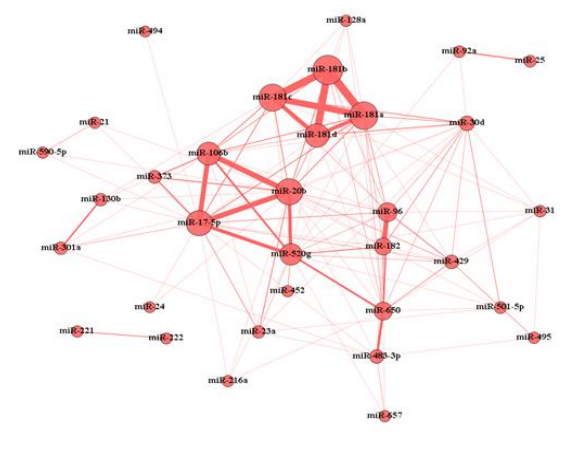

(b)

Fig. 3. Synergistic subnetworks based on different expression profile of miRNAs: (a) synergistic enhancement among down-regulated miRNAs and (b) synergistic suppression among up-regulated miRNAs.

Table 2. Information from Two Different Synergistic Subnetworks

\begin{tabular}{|c|c|c|c|c|c|}
\hline Subnetworks & No. of node & $\begin{array}{l}\text { Average strength of } \\
\text { node }\end{array}$ & Hub miRNAs & No.of pathways & $\begin{array}{l}\text { Top } 5 \text { pathways } \\
\text { (KEGG ID) }\end{array}$ \\
\hline \multirow{5}{*}{$\begin{array}{l}\text { Synergistic } \\
\text { enhancement }\end{array}$} & \multirow{5}{*}{71} & \multirow{5}{*}{58.1} & \multirow{5}{*}{$\begin{array}{l}\text { let-7c, miR-203, } \\
\text { let-7g, let-7i, let-7f, } \\
\text { let-7e }\end{array}$} & \multirow{5}{*}{87} & hsa05200 \\
\hline & & & & & hsa04510 \\
\hline & & & & & hsa04144 \\
\hline & & & & & hsa04010 \\
\hline & & & & & hsa04360 \\
\hline \multirow{5}{*}{$\begin{array}{l}\text { Synergistic } \\
\text { suppression }\end{array}$} & \multirow{5}{*}{33} & \multirow{5}{*}{36.7} & \multirow{5}{*}{$\begin{array}{l}\text { miR-181b, miR-181a, } \\
\text { miR-181c, miR-20b, } \\
\text { miR-17-5p, } \\
\text { miR-181d }\end{array}$} & \multirow{5}{*}{75} & hsa05200 \\
\hline & & & & & hsa04010 \\
\hline & & & & & hsa04510 \\
\hline & & & & & hsa04360 \\
\hline & & & & & hsa04722 \\
\hline
\end{tabular}

The let 7 family be widely reported to be down-regulated significantly in the carcinogenesis of HCC [22], 
and our study confirms the disease relevance but also clarifies its dominating position in miRNA synergism. MiR-29 family serves to reduce cell proliferation and the expression level of miR-29 family are inversely correlated with epithelial-mesenchymal transition status of carcinomas [23]. On the other hand, there has been considerable interest in understanding the roles of miR-181 family in cancer. Upregulation of miR-181b/d at early-stage of NASH-associated hetpatocarcinogenesis indicates their role in disease developing to neoplastic transformation of hepatocytes [24]. Pathways enriched between miR-181 family particularly concentrate in TGF-beta signaling pathway, ErbB signaling pathway and GnRH signaling pathway, whereas synergistic functions between miR-181 family and other non-family miRNAs specially focus on Focal adhesion, MAPK signaling pathway and Wnt signaling pathway.

The top 5 pathways involved in synergistic suppression and enhancement subnetworks have been summarized. Interestingly, four out of the five pathways are common, which means that both synergistic suppression and stimulation on these pathways are significant. It also suggests the complicate influence of miRNAs on pathways. The exact synergistic consequences of miRNA on pathways remain to be fully elucidated by more experimental studies and computer algorithm analysis.

\subsection{Functional Pathway Based on Subnetwork Analysis}

As the complexity of synergistic influence of miRNAs on pathways, we performed a primary investigation to explore the synergistic mechanism of miRNAs by pathway-based subnetwork analysis (Fig. 4). We extract four pivotal pathways from the total MFSN to visualize miRNA synergistic profile on specific pathways, including Focal adhesion (Fig. 4(a)), MAPK signaling pathway (Fig. 4(b)), Wnt signaling pathway (Fig. 4(c)), and TGF-beta signaling pathway (Fig. 4(d)). Meanwhile, the basic information can be found in Table 3.

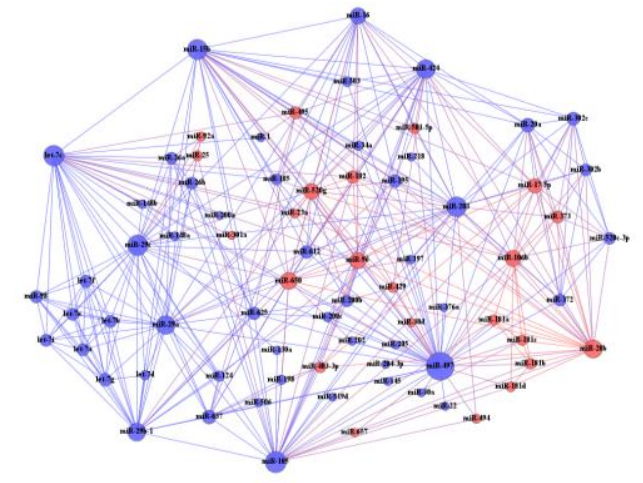

(a)

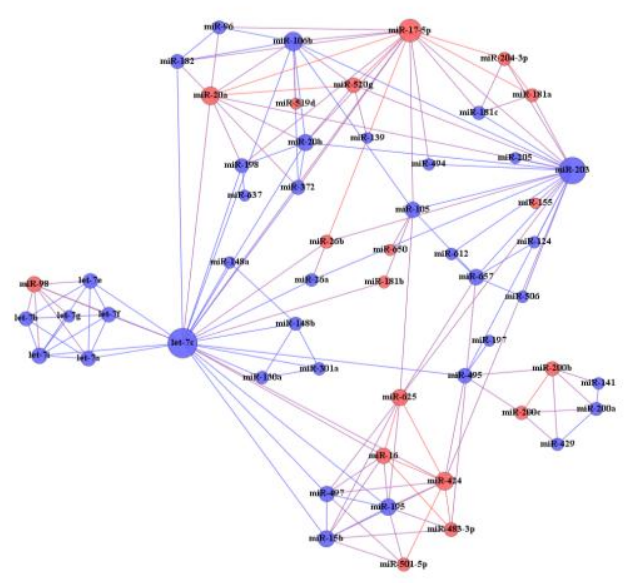

(c)

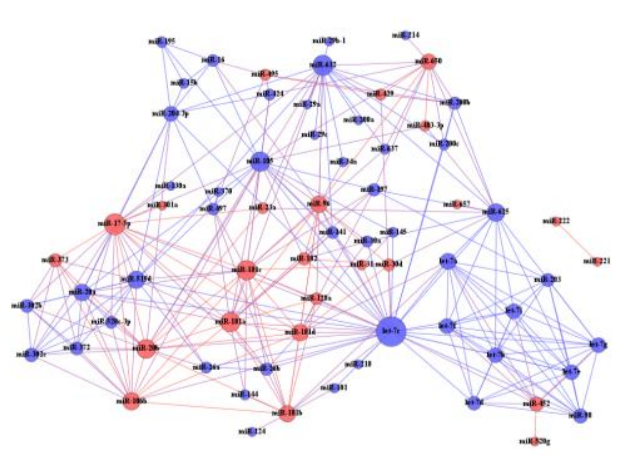

(b)

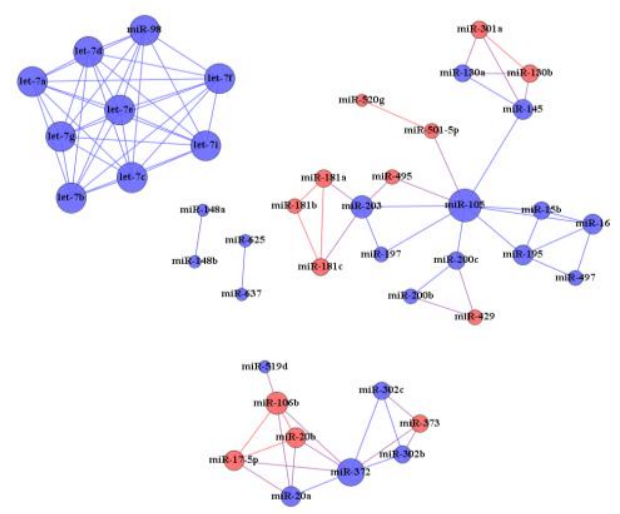

(d)

Fig. 4. Subnetworks related to pivotal pathways extracted from MFSN: (a) Focal adhesion; (b) MAPK signaling pathway; (c) Wnt signaling pathway; (d) TGF-beta signaling pathway. 
As shown in Fig. 4, different pathways involve different number of miRNAs, and each subnetwork owns distinct topological characteristics. Focal adhesion and MAPK signaling pathway show denser network landscapes than the other two pathways, which can be told from average degree of node in subnetworks. Distinct network performances further indicate different synergistic role of DE-miRNAs. Six down-regulated miRNAs locate in prominent positions, leaded by miR-479. Likewise, in TGF-beta signaling pathway, the central positions are also taken by tumor suppressor miRNAs, including miR-105 and let-7 family. Especially, synergistic effect on TGF-beta signaling of let-7 family has been limited in family members, generating an isolated loop in synergistic network. However, both up- and down-regulated miRNAs play key roles in synergistic network related to MAPK signaling pathway and Wnt signaling pathway. However, the ratio of down-regulated miRNAs to up-regulated miRNAs of the four pathways is similar and close to the ratio of total MFSN. Additionally, it is worth of further investigation of the significance caused by the ratio difference.

Table 3. Topologic Information of Four Functional Pathway Based Subnetworks

\begin{tabular}{|c|c|c|c|}
\hline Subnetworks & No. of node & Average degree of node & Hub miRNAs \\
\hline Focal adhesion & 75 & 10 & $\begin{array}{l}\text { miR-497(down), miR-105(down), } \\
\text { let-7c(down), miR-29c(down), } \\
\text { miR-15b(down), miR-203(down) }\end{array}$ \\
\hline MAPK signaling pathway & 70 & 7.9 & $\begin{array}{l}\text { let-7c(down), miR-17-5p(up), } \\
\text { miR-612(down), miR-105(down), } \\
\text { miR-181c(up), miR-181a(up) }\end{array}$ \\
\hline Wnt signaling pathway & 55 & 5.4 & $\begin{array}{l}\text { let-7c(down), miR-203(down), } \\
\text { miR-17-5p(up), miR-106b(down), } \\
\text { miR-20a(up), miR-424(up) }\end{array}$ \\
\hline TGF-beta signaling pathway & 42 & 4.1 & $\begin{array}{l}\text { miR-105(down), let-7 family(let-7a, let-7b, } \\
\text { let-7c, let-7d, let-7e, let-7f, let-7g, let-7i, } \\
\text { miR-98; down) }\end{array}$ \\
\hline
\end{tabular}

\section{Conclusion}

This research analyzes the characteristics of miRNA synergism related to HCC and identifies some active synergistic miRNAs/miRNA families and pivotal pathways, based on the construction and analysis of MFSN, synergistic function based subnetworks, and pathway-based subnetworks. Despite some limitations, this research still provides a systematic insight into miRNA synergism in HCC and further contributes to relevant potential clinical utility.

\section{Acknowledgment}

The authors would like to thank Ms. Tianhong Yin for her helpful comments and suggestions and financial support of the University of Macau by the project, MYRG2015-00172-ICMS-QRCM.

\section{References}

[1] Jemal, A., Bray, F., Center, M. M., Ferlay, J., Ward, E., \& Forman, D. (2011). Global cancer statistics. CA: $A$ Cancer Journal for Clinicians, 61(2), 69-90.

[2] Nordenstedt, H., White, D. L., \& El-Serag, H. B. (2010). The changing pattern of epidemiology in hepatocellular carcinoma. Digestive and Liver Disease, 42, S206-S214.

[3] Sanyal, A. J., Yoon, S. K., \& Lencioni, R. (2010). The etiology of hepatocellular carcinoma and consequences for treatment. The oncologist, 15(Suppl 4), 14-22.

[4] Ambros, V. (2004). The functions of animal microRNAs. Nature, 431(7006), 350-355. 
[5] Hao, Y. X., Wang, J. P., \& Zhao, L. F. (2013). Associations between three common MicroRNA polymorphisms and hepatocellular carcinoma risk in Chinese. Asian Pacific Journal of Cancer Prevention, 14(11), 6601-6604.

[6] Esquela-Kerscher, A., \& Slack, F. J. (2006). Oncomirs-microRNAs with a role in cancer. Nature Reviews Cancer, 6(4), 259-269.

[7] Liu, C., Yu, J., Yu, S., Lavker, R. M., Cai, L., Liu, W., et al. (2010). MicroRNA-21 acts as an oncomir through multiple targets in human hepatocellular carcinoma. Journal of Hepatology, 53(1), 98-107.

[8] Chung, G. E., Yoon, J. H., Myung, S. J., Lee, J. H., Lee, S. H., Lee, S. M., et al. (2010). High expression of microRNA-15b predicts a low risk of tumor recurrence following curative resection of hepatocellular carcinoma. Oncology Reports, 23(1), 113-119.

[9] Aravalli, R. N. (2013). Development of microRNA therapeutics for hepatocellular carcinoma. Diagnostics, 3(1), 170-191.

[10] Li, N., Fu, H., Tie, Y., Hu, Z., Kong, W., Wu, Y., et al. (2009). miR-34a inhibits migration and invasion by down-regulation of c-Met expression in human hepatocellular carcinoma cells. Cancer Letters, 275(1), 44-53.

[11] Yang, J., Han, S., Huang, W., Chen, T., Liu, Y., Pan, S., et al. (2014). A Meta-Analysis of MicroRNA Expression in Liver Cancer. PloS One, 9(12), e114533.

[12] Xu, J., Li, C. X., Li, Y. S., Lv, J. Y., Ma, Y., Shao, T. T., et al. (2011). miRNA-miRNA synergistic network: construction via co-regulating functional modules and disease miRNA topological features. Nucleic Acids Research, 39(3), 825-836.

[13] Zhao, X., Song, H., Zuo, Z., Zhu, Y., Dong, X., \& Lu, X. (2013). Identification of miRNA-miRNA synergistic relationships in colorectal cancer. International Journal of Biological Macromolecules, 55, 98-103.

[14] Ying, H., Lyu, J., Ying, T., Li, J., Jin, S., Shao, J., et al. (2014). Risk miRNA screening of ovarian cancer based on miRNA functional synergistic network. Journal of Ovarian Research, 7(1), 9.

[15] Xiao, S., Ma, Y., Zhu, H., Sun, H., Yin, Y., \& Feng, G. (2015). miRNA functional synergistic network analysis of mice with ischemic stroke. Neurological Sciences, 36(1), 143-148.

[16] Xie, B., Ding, Q., Han, H., \& Wu, D. (2013). miRCancer: A microRNA-cancer association database constructed by text mining on literature. Bioinformatics, btt014.

[17] Xiao, F., Zuo, Z., Cai, G., Kang, S., Gao, X., \& Li, T. (2009). miRecords: An integrated resource for microRNA-target interactions. Nucleic Acids Research, 37(suppl 1), D105-D110.

[18] Yu, G., Wang, L. G., Han, Y., \& He, Q. Y. (2012). Clusterprofiler: An R package for comparing biological themes among gene clusters. Omics: A Journal of Integrative Biology, 16(5), 284-287.

[19] Bastian, M., Heymann, S., \& Jacomy, M. (2009). Gephi: An open source software for exploring and manipulating networks. ICWSM, 8, 361-362.

[20] Tsang, J. S., Ebert, M. S., \& van Oudenaarden, A. (2010). Genome-wide dissection of microRNA functions and cotargeting networks using gene set signatures. Molecular Cell, 38(1), 140-153.

[21] Garzon, R., Marcucci, G., \& Croce, C. M. (2010). Targeting microRNAs in cancer: Rationale, strategies and challenges. Nature Reviews Drug Discovery, 9(10), 775-789.

[22] Boyerinas, B., Park, S. M., Hau, A., Murmann, A. E., \& Peter, M. E. (2010). The role of let-7 in cell differentiation and cancer. Endocrine-Related Cancer, 17(1), F19-F36.

[23] Garzon, R., Heaphy, C. E., Havelange, V., Fabbri, M., Volinia, S., Tsao, T., et al. (2009). MicroRNA 29b functions in acute myeloid leukemia. Blood, 114(26), 5331-5341.

[24] Wang, B., Hsu, S. H., Majumder, S., Kutay, H., Huang, W., Jacob, S. T., et al. (2010). TGF $\beta$-mediated upregulation of hepatic miR-181b promotes hepatocarcinogenesis by targeting TIMP3. Oncogene, 29(12), 1787-1797. 


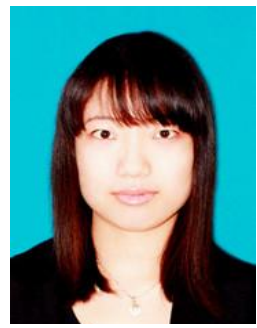

Xiangjun Kong was born in China in 1987. She obtained her B.Eng. with honours in bioengineering (2010) and the M.Sc. with honours in biology science (2013) from the Chongqing University, China.

She is currently a Ph.D student at the Institute of Chinese Medical Sciences, University of Macau. She has published several research papers in leading international journals, such as Expert Opinion on Therapeutic Patents, Human Vaccines \& Immunotherapeutics, and Evidence-Based Complementary and Alternative Medicine. Her research interests are network analysis, patent landscape, bioinformatics, and knowledge flow study.

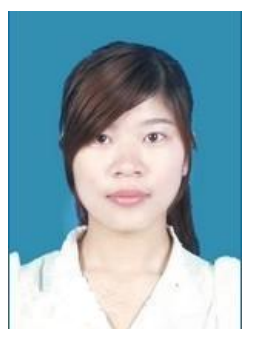

Xiaomei Geng is currently a graduate student of medicinal administration at Institute of Chinese Medical Sciences, University of Macau. She obtained her B.Sc. with honours in biopharmaceutics from Soochow University, China.

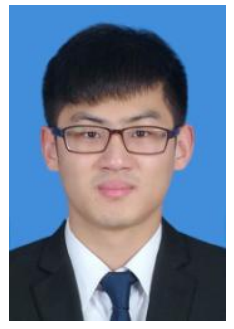

Junrui Zhao is under master's programme of medicinal administration at Institute of Chinese Medical Sciences, University of Macau. He holds his B.Sc. degree in pharmacy from Guangzhou University of Chinese Medicine, China.

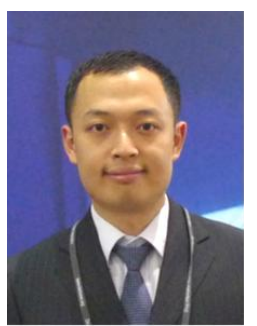

Yuanjia Hu is an assistant professor of State Key Laboratory of Quality Research in Chinese Medicine \& Institute of Chinese Medical Sciences, University of Macau (UM). He has published over 60 academic articles (SCI, SSCI, CSCI and CSSCI, etc). He received the B.Sc. degree from the School of International Pharmaceutical Business, China Pharmaceutical University (CPU), UM-CPU dual M.Sc degree in medicinal administration, and Ph.D. degree in biomedical sciences from the UM, while he studied innovation economics in Austrian Research Centers as a visiting scholar. 